\title{
АНАЛИТИЧЕСКОЕ КОНСТРУИРОВАНИЕ РАЗОМКНУТЫХ СИСТЕМ СТОХАСТИЧЕСКОГО УПРАВЛЕНИЯ МНОГОМЕРНЫМ НЕЛИНЕЙНЫМ ОБЪЕКТОМ
}

Analytical design of the open-loop systems of stochastic multidimensional nonlinear object control.

Блохин Л.Н. ( Blohin, L.N.) ${ }^{1}$, д.т.н., проф., Осадчий С.И. (Osadchiy S.I.) ${ }^{2}$, д.т.н., проф., Зозуля В.A.(Zоzulia V.A.) ${ }^{3}$, к.т.н., доцент

${ }^{1}$ Национальный авиационный университет, г. Киев

${ }^{2,3}$ Кировоградский национальный технический университет, Кировоград

E-mail: ${ }^{2}$ srg2005@ukr.net, ${ }^{3}$ irish38@ukr.net

${ }^{2}$ ORCID: 0000-0002-1811-3594

${ }^{3}$ ORCID: 0000-0003-3793-4686

Copyright (C) 2014 by author and the journal "Automation technological and business - processes". This work is licensed under the Creative Commons Attribution International License (CC BY). http://creativecommons.org/licenses/by/4.0/

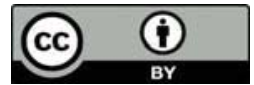

\begin{abstract}
Аннотация
Работа посвящена разработке нового метода синтеза матрицы передаточных функций регулятора оптимальной многомерной разомкнутой системы управления. Регулятор предназначен для максимизации точности перехода нелинейного многомерного объекта управления из одного установившегося режима в другой. Предполагается, что на объект управления действуют возмущения, а датчики измерительной информации обладают инерцией и помехами. Как возмущения, так и помехи представляют собой аддитивную комбинацию регулярных и случайных составляющих. Случайные составляющие ограничены классом взаимосвязанных стационарных процессов с дробно-рациональными матрицами спектральных плотностей. Обоснованный метод отличается тем, что при постановке и решении задачи синтеза используется новая структурная схема системы управления, учитывающая результаты метрологической аттестации динамики датчиков. Синтез регулятора осуществляется в частотной области методом Винера-Колмогорова. Полученный в результате решения задачи синтеза новый алгоритм позволяет найти матрицы передаточных функций регулятора, которые обеспечивают минимум соответствующих квадратичных критериев качества. Первый из них представляет сумму определенным образом взвешенных интегральных квадратичных отклонений регулярных ошибки следования объекта по траектории и сигнала управления. Второй критерий представляет сумму взвешенных дисперсий случайных составляющих ошибки и сигнала управления. Для использования предложенного алгоритма необходио выполнить операции винеровской факторизации и сепарации дробнорациональных матриц. Соответствующие функции содержатся в свободно распространяемом пакете программ SciLab.
\end{abstract}

Ключевые слова

Оптимальный, управление, объект, матрица, факторизация, дисперсия, стохастический

Abstract

This article is devoted to the development of a new method of synthesis a regulators' transfer functions matrix of an optimal multivariable open-loop control system. The regulator is designed to maximize an accuracy of a nonlinear multivariable control object transition from one steady state to another. It is assumed that disturbances act on the control object and sensors measuring data has inertia and noise. Both disturbances and noises are an additive combination of regular and random components. Random components belong to a class of interconnected stationary processes with rational spectral density matrices. A substantiated method differs from the known one by the fact that 
during formulation and solution of the problem somebody uses a new block diagram of the control system, which takes into account the results of metrological certification of a sensor dynamics. Synthesis of the regulator is carried out in the frequency domain by the Wiener-Kolmogorov method. A new algorithm, which is obtained as a result of synthesis problem solution, allows you to find the matrix of regulator transfer functions, which provides a minimum of corresponding quadratic quality criteria's. The first of them is equal to the sum of certain way weighted squared deviations regular repetition errors of the object path and control signals. The second criterion is equal to the sum of the weighted variance of the random error components and the control signals. To execute the proposed algorithm it is necessary to perform the operations of Wiener factorization and separation of rational matrices. The corresponding functions are contained in the freely distributed software package SciLab.

Keywords

Optimal, control, object, matrix, factorization, variance, stochastic.

\section{Введение}

Развитие использования модульного принципа конструирования систем управления многомерными объектами приводит к расширению использования разомкнутых систем управления. Наиболее ярким примером таких систем является управление рабочими органами роботов манипуляторов или программными движениями мехатронных объектов типа гексапод [1].

Как правило, объекты управления этого класса являются многомерными и нелинейными, а их движение происходит в условиях совместного действия аддитивной смеси векторов детерминированных и стационарных центрированных случайных сигналов. Процесс их движения по траектории, как правило, сопровождается изменением динамики объекта управления, а амплитуда управляющего воздействия ограничена. Как показано в монографии [2] управление объектами этого класса сопровождается эффектом самоорганизации [2], который часто приводит к ухудшению точности выполнения программы движения. Следовательно, выполнение исследований направленных на разработку новых алгоритмов проектирования систем управления предотвращающих возникновение самоорганизации является актуальным.

В основу разработки предлагается положить принцип разделения всей траектории движения нелинейного объекта управления на ограниченное число участков и синтеза оптимального по точности регулятора для каждого из выделенных участков.

Анализ литературных данных и постановка проблемы

Анализ целого ряда работ, например [3,4,5], позволяет сделать вывод о том, что современные методы проектирования оптимальных нелинейных систем управления гарантируют успех в двух случаях. Первый - возникает при действии только детерминированных полезных сигналов, возмущений и помех в трактах управления [3], второй при действии только случайных воздействий [4].

Рассмотрению технологий идентификации и синтеза оптимальных систем управления при действии аддитивной смеси векторов регулярных и случайных составляющих полезных сигналов, возмущений и помех в частотной области посвящена монография [5], однако предложенные в ней методы справедливы в случае линейного многомерного объекта управления.

Монография [6] содержит целый ряд методов и подходов к разработке оптимальных многомерных систем управления объектами в пространстве состояний, использование которых, однако, ограничено либо задачей управления, либо классом возмущений, действующих во время управления, либо классом линеаризованной модели объекта управления. Ограничение задачи управления - это отработка начальных условий при отсутствии возмущений и помех. Класс возмущений ограничен их принадлежностью к стационарным многомерным процессам типа «белый шум», а класс линеаризованной модели объекта управления ограничивается только теми объектами, модель динамики которых может быть представлена в виде дифференциальных уравнений в форме Коши.

В реальной ситуации управления сложным многомерным нелинейным динамическим объектом указанные ограничения далеко не всегда выполняются. Наиболее ярким примером такого невыполнения является суперкавитационный подводный объект [7].

Для устранения таких ограничений и как развитие методов, предложенных в работах [3,5], в данной статье ставится и решается задача синтеза оптимальной многомерной разомкнутой системы управления нелинейным динамическим объектом, в условиях действия аддитивной смеси векторов детерминированных и центрированных стационарных случайных полезных сигналов, возмущений и помех.

Постановка задачи

Пусть задана структурная схема разомкнутой системы управления в j-том режиме (рис. 1). Она включает нелинейный объект управления, имеющий $m$ управляемых входов и $n$ выходов, и регулятор. 


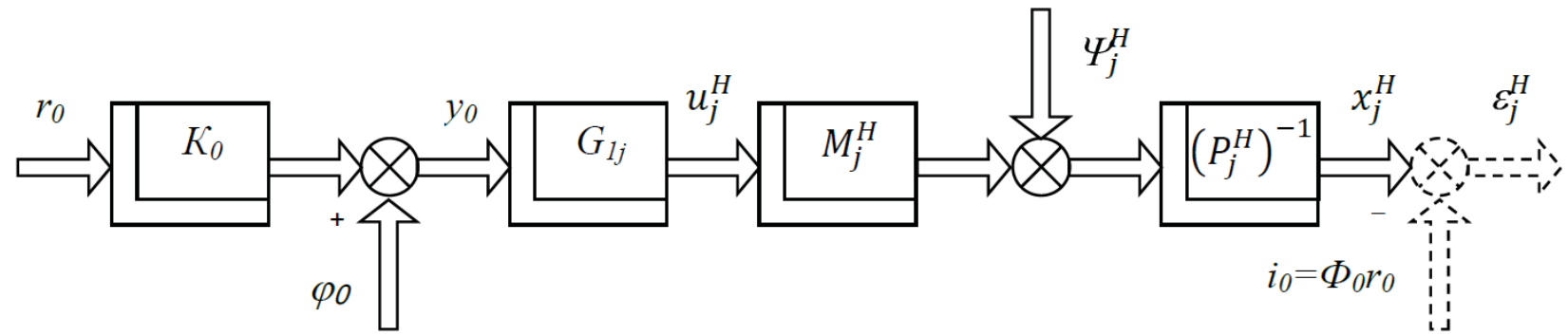

Рис. 1 - Структурная схема разомкнутой системы управления нелинейным объектом в ј-том режиме его функционирования

Объект может функционировать в одном из $k$ режимов, а его динамика в режиме номер $j$ описана линеаризованной системой обыкновенных дифференциальных уравнений вида

$$
P_{j}^{H} x_{j}^{H}=M_{j}^{H} u_{j}^{H}+\Psi_{j}^{H} g,
$$

где $P_{j}^{H}$ - полиномиальная матрица размерности $n \times n$, элементами которой являются полиномы от оператора

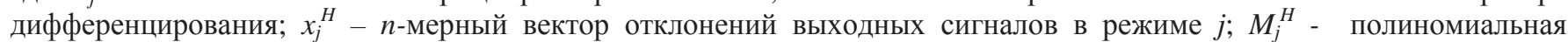
матрица размерности $m \times n ; u_{j}^{H}-m$-мерный вектор отклонений сигналов управления; $\Psi_{j}^{H}-$ полиномиальная матрица размерности $n \times n$, которая характеризует влияние возмущений на вектор выходных координат объекта; $g-n$-мерный вектор типовых воздействий. Если левую и правую части системы уравнений (1) помножить на обратную к $P_{j}^{H}$ матрицу $\left(P_{j}^{H}\right)^{-1}$, то при нулевых начальных условиях формируется к-мерный набор моделей динамики векторов выходных сигналов объекта вида

$$
\begin{aligned}
& x_{1}^{H}=V_{1}^{H} \cdot\left(\begin{array}{c}
u_{1}^{H} \\
g
\end{array}\right) ; \\
& x_{2}^{H}=V_{2}^{H} \cdot\left(\begin{array}{c}
u_{2}^{H} \\
g
\end{array}\right) ; \\
& \ldots \ldots \ldots \ldots \ldots \ldots . . \\
& x_{j}^{H}=V_{j \cdot}^{H} \cdot\left(\begin{array}{c}
u_{j}^{H} \\
g
\end{array}\right) ; \\
& \ldots \ldots \ldots \ldots \ldots . . \\
& x_{k}^{H}=V_{k}^{H} \cdot\left(\begin{array}{c}
u_{k}^{H} \\
g
\end{array}\right),
\end{aligned}
$$

где $V_{j}^{H}$ - блочная матрица передаточных функций расширенного объекта управления в режиме функционирования номер $j$, которая равна

$$
V_{j}^{H}=\left(\Phi_{1 j}^{H}, \Phi_{2 j}^{H}\right)=\left[\left(P_{j}^{H}\right)^{-1} M_{j}^{H},\left(P_{j}^{H}\right)^{-1} \Psi_{j}^{H}\right] .
$$

Регулятор также может функционировать в одном из $k$ режимов. На его входах действуют вектор программных сигналов $r_{0}$ и вектор помех задания программы $\varphi_{0}$, а на выходе формируется вектор сигналов управления $u_{j}^{H}$. Динамику регулятора характеризуют матрицы передаточных функций задатчика программы $K_{0}$ и звена коррекции управления в режиме номер $j G_{l j}$.

Качество работы системы (рис. 1) оценивается вектором ошибок управления в каждом из режимов работы $\varepsilon_{j}^{H}$, который характеризует отклонение вектора отклонений выходных сигналов от вектора желаемых сигналов $i_{0}$

$$
\varepsilon_{j}^{H}=x_{j}^{H}-i_{0}=x_{j}^{H}-\Phi_{0} \mathrm{r}_{0},
$$

где $\Phi_{0}$ - матрица передаточных функций желаемого преобразования вектора программы соответствующей размерности.

Очевидно, что приближение вектора $y_{0}$ к вектору $r_{0}$ и соответствующий выбор матрицы передаточных функций $G_{l j}$ позволяют уменьшать вектор ошибок управления (4).

Для формулирования задачи синтеза оптимальной структуры регулятора в разомкнутой системе управления целесообразно модернизировать тракт задания вектора программных сигналов как показано на рис. 2. 


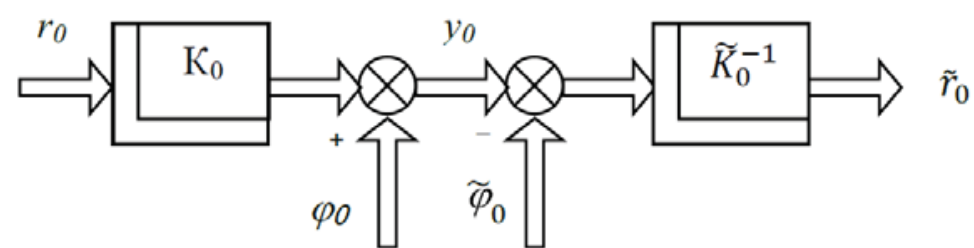

Рис. 2 - Структурная схема модернизированного тракта задания программы движения

Тогда на вход звена коррекции управления будет подаваться вектор $\tilde{r}_{0}$, который формируется в результате решения следующего уравнения

$$
\tilde{r}_{0}=\tilde{K}_{0}^{-1}\left(K_{0} r_{0}+\varphi_{0}-\tilde{\varphi}_{0}\right) \approx r_{0}+\tilde{K}_{0}^{-1} \theta_{\varphi_{0}},
$$

где индекс « » над векторами и передаточными функциями звеньев отражает факт получения указанных характеристик по результатам специальных испытаний, а вектор $\theta_{\varphi 0}$ полагается малым. Следовательно, вектор сигналов управления $u_{j}^{H}$ будет равен

$$
u_{j}^{H}=G_{j 1} \tilde{r}_{0} .
$$

Учитывая выражения (2), (3) и (5), а также структурные схемы рис.1, рис. 2, частотная характеристика вектора выходных сигналов в режиме $j$ представляется в виде

$$
x_{j}^{H}=V_{j}^{H} \cdot\left(\begin{array}{c}
G_{j 1}\left[r_{0}+\tilde{K}_{0}^{-1} \theta_{\varphi 0}\right] \\
g
\end{array}\right) .
$$

Подстановка выражения (6) в уравнение (4) позволяет определить вид вектора ошибок управления $\varepsilon_{j}^{H}$ и эрмитово сопряженного к нему вектора следующим образом

$$
\begin{gathered}
\varepsilon_{j}^{H}=x_{j}^{H}-i_{0}=\Phi_{1 j}^{H} G_{j 1}\left(r_{0}+\tilde{K}_{0}^{-1} \theta_{\varphi_{0}}\right)+\Phi_{2 j}^{H} g-\Phi_{0} r_{0}, \\
\varepsilon_{j^{*}}^{H}=\left(r_{0^{*}}+\tilde{K}_{0^{*}}^{-1} \theta_{\varphi_{0^{*}}}\right) G_{j 1^{*}} \Phi_{1 j^{*}}^{H}+g_{*} \Phi_{2 j^{*}}^{H}-r_{0^{*}} \Phi_{0^{*}},
\end{gathered}
$$

здесь нижний индекс * означает операцию эрмитового сопряжения вектора или матрицы [9].

Будем считать также, что в процессе функционирования на систему действуют стохастические векторы программных сигналов, возмущений и помех, которые представляют аддитивную смесь детерминированной и стационарной центрированной случайной составляющих. В таких условиях функционал качества управления объектом в ј-том режиме его функционирования при детерминированных входных воздействиях должен быть таким

$$
I_{j}^{H}=\frac{1}{2 \pi j} \int_{-j \infty}^{j \infty} \operatorname{tr}\left(\bar{\varepsilon}_{j}^{H} \cdot \bar{\varepsilon}_{j^{*}}^{H} \bar{R}_{j}\right) d s, \quad s=j \omega,
$$

а при стационарных случайных воздействиях должен быть следующим

$$
\dot{e}_{j}^{H}=\frac{1}{j} \int_{-j \infty}^{j \infty} \operatorname{tr}\left(S_{\varepsilon \varepsilon}^{\prime} \dot{R}_{j}\right) d s,
$$

где $j$ - комплексная единица, tr - знак операции поиска седа матрицы [9]; черта над вектором - символ детерминированной составляющей вектора; $S_{\varepsilon \varepsilon}{ }_{\varepsilon \varepsilon}$ - транспонированная матрица спектральных плотностей случайной составляющей вектора ошибок управления (7); $\bar{R}_{j}$ и $\dot{R}_{j}$ - весовые положительно-определенные матрицы. По теореме Винера-Хинчина [10] транспонированная матрица $S_{\varepsilon \varepsilon}{ }_{\varepsilon \varepsilon}$ связана с матрицами спектральных плотностей случайных составляющих воздействий $r_{0}, \theta_{\varphi 0}$ и $g$ уравнением

$$
\begin{gathered}
S_{\varepsilon \varepsilon}^{\prime}=\Phi_{1 \mathrm{j}}^{\mathrm{H}} G_{j 1^{s}}^{s} S_{r r}^{\prime} G_{j 1^{*}}^{s} \Phi_{1 \mathrm{j}}^{H}+\Phi_{1 j}^{H} G_{j 1}^{s} \tilde{K}_{0}^{-1} S_{r \theta}^{\prime} G_{j 1^{*}}^{s} \Phi_{1 j^{*}}^{H}+\Phi_{1 j}^{H} G_{j 1}^{s} S_{\theta r}^{\prime} \tilde{K}_{0^{*}}^{-1} G_{j 1^{*}}^{s} \Phi_{1 j^{*}}^{H}+\Phi_{1 j}^{H} G_{j 1}^{s} \tilde{K}_{0}^{-1} S_{\theta \theta}^{\prime} \tilde{K}_{0^{*}}^{-1} G_{j 1^{*}}^{s} \Phi_{1 j^{*}}^{H}+ \\
+\Phi_{1 \mathrm{j}}^{\mathrm{H}} G_{j 1}^{s} S_{\Delta r}^{\prime} \Phi_{2 j^{*}}^{H}+\Phi_{1 j}^{H} G_{j 1}^{s} \tilde{K}_{0}^{-1} S_{\Delta \theta}^{\prime} \Phi_{2 j^{*}}^{H}-\Phi_{1 j}^{H} G_{j 1}^{s} S_{r r}^{\prime} \tilde{K}_{0^{*}}^{-1} \Phi_{0^{*}}^{s}-\Phi_{1 j}^{H} G_{j 1}^{s} \tilde{K}_{0}^{-1} S_{r \theta}^{\prime} \Phi_{0^{*}}^{s}+\Phi_{2 j}^{H} S_{\Delta \Delta}^{\prime} \Phi_{2 j^{*}}^{H}-\Phi_{2 j}^{H} S_{r \Delta}^{\prime} \Phi_{0^{*}}^{s}- \\
-\Phi_{0}^{\mathrm{s}} S_{r r}^{\prime} G_{j 1^{*}}^{s} \Phi_{1 j^{*}}^{H}-\Phi_{0}^{s} S_{\theta r}^{\prime} \tilde{K}_{0^{*}}^{-1} G_{j 1^{*}}^{s} \Phi_{1 j^{*}}^{H}-\Phi_{0}^{s} S_{\Delta r}^{\prime} \Phi_{2 j^{*}}^{H}+\Phi_{0}^{s} S_{r r}^{\prime} \Phi_{0^{*}}^{s},
\end{gathered}
$$

где $S_{r r}$ ' - транспонированная матрица спектральных плотностей случайной составляющей вектора программных сигналов $r_{0} ; S_{r \theta}^{\prime}$ - транспонированная матрица взаимных спектральных плотностей между случайными составляющими векторов $r_{0}$ и $\theta_{\varphi 0} ; S_{\theta \theta}^{\prime}$ - транспонированная матрица спектральных плотностей случайной составляющей вектора $\theta_{\varphi 0} ; S_{\Delta r}{ }^{\prime}$ - транспонированная матрица взаимных спектральных плотностей между случайными составляющими векторов $\Delta$ и $r_{0} ; S_{\Delta \theta}^{\prime}$ - транспонированная матрица взаимных спектральных плотностей между случайными составляющими векторов $g$ и $\theta ; G_{j l}{ }^{s}-$ матрица передаточных функций звена коррекции случайной составляющей управления.

Таким образом, задача синтеза состоит в том, чтобы по известному набору моделей динамики объекта управления (2), векторов программных сигналов $r_{0}$, возмущений $g$ и помех $\theta_{\varphi 0}$ обосновать процедуру поиска 
структуры и параметров матриц передаточных функций детерминированного $\left(G_{j 1}^{d}\right)$ и случайного $\left(G_{j 1}^{s}\right)$ трактов регулятора, которые в каждом из возможных режимов функционирования доставят минимум функционалам качества (8), (9).

Учитывая результаты, полученные в монографиях $[5,10]$, решение поставленной задачи сводится к разработке двух алгоритмов синтеза матриц передаточных функций регулятора.

А. Синтез оптимальной структуры матрицы передаточных функций $G^{\mathrm{d}} 1$ регулятора в системе управления нелинейным объектом в $\boldsymbol{j}$-том режиме функционирования при детерминированных воздействиях

Если в уравнение (7) вместо матрицы передаточных функций $G_{j l}$ подставить матрицу передаточных функций детерминированного тракта регулятора $G_{\mathrm{j} 1}{ }^{d}$, а вместо матрицы желаемых преобразований $\Phi_{0}-$ матрицу передаточных функций желаемого преобразования детерминированной составляющей программного сигнала $\Phi_{0}{ }^{d}$ то функционал качества (8) представляется в виде

$$
\begin{gathered}
I_{j}^{H}=\frac{1}{2 \pi j} \int_{-j \infty}^{j \infty} \operatorname{tr}\left[\Phi_{1 j}^{\mathrm{H}} G_{j 1}^{d} \bar{r}_{0} \bar{r}_{0^{*}} G_{j 1^{*}}^{d} \Phi_{1 \mathrm{j}}^{H}+\Phi_{1 j}^{H} G_{j 1}^{d} \tilde{K}_{0}^{-1} \bar{\theta}_{\varphi 0} \bar{r}_{0^{*}} G_{j 1^{*}}^{d} \Phi_{1 j^{*}}^{H}+\Phi_{1 j}^{H} G_{j 1}^{d} \bar{r}_{0} \bar{\theta}_{\varphi 0^{*}} \tilde{K}_{0^{*}}^{-1} G_{j 1^{*}}^{d} \Phi_{1 j^{*}}^{H}+\right. \\
+\Phi_{1 j}^{H} G_{j 1}^{d} \tilde{K}_{0}^{-1} \bar{\theta}_{\varphi 0} \bar{\theta}_{\varphi 0^{*}} \tilde{K}_{0^{*}}^{-1} G_{j 1^{*}}^{d} \Phi_{1 j^{*}}^{H}+\Phi_{1 j}^{\mathrm{H}} G_{j 1}^{d} \bar{r}_{0} g_{*} \Phi_{2 j^{*}}^{H}+\Phi_{1 j}^{H} G_{j 1}^{d} \tilde{K}_{0}^{-1} \bar{\theta}_{\varphi 0} g_{*} \Phi_{2 j^{*}}^{H}-\Phi_{1 j}^{H} G_{j 1^{\prime}}^{d} \bar{r}_{0^{*}} \bar{r}_{0^{*}} \tilde{K}_{0^{*}}^{-1} \Phi_{0^{*}}^{d}- \\
-\Phi_{1 j}^{H} G_{j 1}^{d} \tilde{K}_{0}^{-1} \bar{\theta}_{\varphi 0} \bar{r}_{0^{*}} \Phi_{0^{*}}^{d}+\Phi_{2 j}^{H} g g_{*} \Phi_{2 j^{*}}^{H}-\Phi_{2 j}^{H} g \bar{r}_{0^{*}} \Phi_{0^{*}}^{d}-\Phi_{0}^{\mathrm{d}} \bar{r}_{0} \bar{r}_{0^{*}} G_{j 1^{*}}^{d} \Phi_{1^{*}}^{H}-\Phi_{0}^{d} \bar{r}_{0} \bar{\theta}_{\varphi 0} \tilde{K}_{0^{*}}^{-1} G_{j 1^{*}}^{d} \Phi_{1 j^{*}}^{H}-\Phi_{0}^{d} \bar{r}_{0} g_{*} \Phi_{2 j^{*}}^{H}+ \\
\left.+\Phi_{0}^{d} \bar{r}_{0} \bar{r}_{0^{*}} \Phi_{0^{*}}^{d}\right] \bar{R}_{j} d s,
\end{gathered}
$$

а задача синтеза сводится к тому, чтобы по известным матрицам передаточных функций $\Phi_{1 j}{ }^{\mathrm{H}}, K_{0}, \Phi_{0}{ }^{d}$ и векторам частотных характеристик детерминированных составляющих сигналов $\overline{r_{0}}, g, \theta_{\varphi 0}$ найти уравнение для расчета такой физически реализуемой и устойчивой матрицы передаточных функций $G_{\mathrm{j} 1}{ }^{d}$, при которой функционал (11) достигает минимума.

Для решения поставленной задачи использован метод Винера-Колмогорова, в соответствии с которым необходимо найти условие равенства нулю первой вариации функционала (11) на классе устойчивых и физически реализуемых варьируемых матриц $G_{\mathrm{j} 1}{ }^{d}$.

Первая вариация функционала (11) имеет вид

$$
\begin{gathered}
\delta I_{j}^{H}=\frac{1}{2 \pi j} \int_{-j \infty 0}^{j \infty} \operatorname{tr}\left\{\Phi_{1 j^{*}}^{\mathrm{H}} \bar{R}_{j} \Phi_{1 \mathrm{j}}^{H} G_{j 1}^{d}\left(\bar{r}_{0} \bar{r}_{0^{*}}+\tilde{K}_{0}^{-1} \bar{\theta}_{\varphi 0} \bar{r}_{0^{*}}+\bar{r}_{0} \bar{\theta}_{\varphi 0^{*}} \tilde{K}_{0^{*}}^{-1}+\tilde{K}_{0}^{-1} \bar{\theta}_{\varphi 0} \bar{\theta}_{\varphi 0^{*}} K_{0^{*}}^{-1}\right)+\Phi_{1 j^{*}}^{H} \bar{R}_{j}\left(\Phi_{2 j}^{H} g \bar{r}_{0^{*}}+\Phi_{2 j}^{H} g \bar{\theta}_{\varphi 0^{*}} \tilde{K}_{0^{*}}^{-1}-\right.\right. \\
\left.\left.-\Phi_{0}^{d} \bar{r}_{0} \bar{r}_{0^{*}}-\Phi_{0}^{d} \bar{r}_{0} \bar{\theta}_{\varphi 0^{*}} \tilde{K}_{0^{*}}^{-1}\right)\right] \delta G_{j 1^{*}}^{d}+ \\
\delta G_{j 1}^{d}\left[\left(\bar{r}_{0} \bar{r}_{0^{*}}+\tilde{K}_{0}^{-1} \bar{\theta}_{\varphi 0} \bar{r}_{0^{*}}+\bar{r}_{0} \bar{\theta}_{\varphi 0^{*}} \tilde{K}_{0^{*}}^{-1}+\tilde{K}_{0}^{-1} \bar{\theta}_{\varphi 0} \bar{\theta}_{\varphi 0^{*}} \tilde{K}_{0^{*}}^{-1}\right) G_{j 1^{*}}^{d} \Phi_{1 j^{*}}^{H} \bar{R}_{j} \Phi_{1 j}^{H}+\left(\bar{r}_{0} g_{*} \Phi_{2 j^{*}}^{H}+\right.\right. \\
\left.\left.\left.+\tilde{K}_{0}^{-1} \bar{\theta}_{\varphi 0} g_{*} \Phi_{2 j^{*}}^{H}-\bar{r}_{0} \bar{r}_{0^{*}} \Phi_{0^{*}}^{d}-\tilde{K}_{0}^{-1} \bar{\theta}_{\varphi 0^{*}} \bar{r}_{0^{*}} \Phi_{0^{*}}^{d}\right) \bar{R}_{j} \Phi_{1 j}^{H}\right]\right\} d s .
\end{gathered}
$$

Если в результате винеровской факторизации [11] найти дробно-рациональную матрицу $\Gamma_{j}^{d}$, устойчивую вместе с обратной и такую, что

$$
\Gamma_{j^{*}}^{d} \Gamma_{j}^{d}=\Phi_{1 j^{*}}^{H} \bar{R}_{j} \Phi_{1 j}^{H},
$$

в результате приближенной факторизации найти дробно-рациональную матрицу $D_{j}^{\mathrm{d}}$ со свойствами аналогичными матрице $\Gamma_{j}^{d}$ при которой выполняется равенство

$$
D_{j}^{d} D_{j^{*}}^{d}=\bar{r}_{0} \bar{r}_{0^{*}}+\tilde{K}_{0}^{-1} \bar{\theta}_{\varphi 0} \bar{r}_{0^{*}}+\bar{r}_{0} \bar{\theta}_{\varphi 0^{*}} \tilde{K}_{0^{*}}^{-1}+\tilde{K}_{0}^{-1} \bar{\theta}_{\varphi 0} \bar{\theta}_{\varphi 0^{*}} \tilde{K}_{0^{*}}^{-1}
$$

а также выполнить винеровскую сепарацию [10] матрицы $T_{\mathrm{j}}^{\mathrm{d}}$ вида

$$
T_{j}^{d}=T_{j 0}^{d}+T_{j+}^{d}+T_{j-}^{d}=\left(\Gamma_{j^{*}}^{d}\right)^{-1} \Phi_{1 j^{*}}^{H} \bar{R}_{j}\left(\Phi_{2 j}^{H} g \bar{r}_{0^{*}}+\Phi_{2 j}^{H} g \bar{\theta}_{\varphi 0^{*}} \tilde{K}_{0^{*}}^{-1}-\Phi_{0}^{d} \bar{r}_{0} \bar{r}_{0^{*}}-\Phi_{0}^{d} \bar{r}_{0} \bar{\theta}_{\varphi 0^{*}} \tilde{K}_{0^{*}}^{-1}\right)\left(D_{j^{*}}^{d}\right)^{-1},
$$

то функционал (12) можно переписать так:

$$
\delta I_{j}^{H}=\frac{1}{2 \pi j} \int_{-j \infty}^{j \infty} \operatorname{tr}\left[\Gamma_{j^{*}}^{d}\left(\Gamma_{j}^{d} G_{j 1}^{d} D_{j}^{d}-T_{j}^{d}\right) D_{j^{*}}^{d} \delta G_{j 1^{*}}^{d}+\delta G_{j 1}^{d} D_{j}^{d}\left(D_{j^{*}}^{d} G_{j 1^{*}}^{d} \Gamma_{j^{*}}^{d}-T_{j^{*}}^{d}\right) \Gamma_{j}^{d}\right] d s .
$$

Условия равенства нулю первой вариации (16) на классе устойчивых и физически реализуемых матриц передаточных функций $G_{\mathrm{j} 1}{ }^{d}$ по аналогии с соотвествующим результатом из монографий $[5,10]$ имеет вид

$$
\Gamma_{j}^{d} G_{j 1}^{d} D_{j}^{d} \approx\left(T_{j 0}^{d}+T_{j+}^{d}\right),
$$

тогда уравнение для расчета такой физически реализуемой и устойчивой матрицы передаточных функций $G_{\mathrm{j} 1}{ }^{d}$ представляется так

$$
G_{j 1}^{d} \approx\left(\Gamma_{j}^{d}\right)^{-1}\left(T_{j 0}^{d}+T_{j+}^{d}\right)\left(D_{j}^{d}\right)^{-1}
$$

После синтеза структуры $G_{\mathrm{j} 1}{ }^{d}$ необходимо определить частотную характеристику нового вектора сигналов управления

$$
\bar{u}^{H}=\bar{G}_{j 1}^{d} \cdot\left(\bar{r}_{0}+\tilde{K}_{0}^{-1} \bar{\theta}_{\varphi 0}\right)
$$


и сравнить ее с заданными частотными характеристиками набора векторов управления. В результате сравнения необходимо найти номер $j$ наиболее близкого к вектору (18) вектора сигналов управления. После установления номера вектора сигналов управления приведенная выше процедура синтеза структуры нового звена коррекции должна быть повторена с использованием полученного номера режима.

Таким образом задача синтеза оптимальной структуры передаточной функции звена коррекции при детерминированных сигналах решена.

\section{Б. Синтез структуры регулятора $\dot{G}_{2}^{H}$ в системе управления нелинейным объектом при стационарных} случайных воздействиях

Задача синтеза матрицы передаточных функций звена коррекции при случайных воздействиях состоит в том, чтобы по известным матрицам передаточных функций $\Phi_{1 j}{ }^{\mathrm{H}}, K_{0}, \Phi_{0}{ }^{d}$ и транспонированным матрицам спектральных и взаимных спектральных плотностей $S_{r r}{ }^{\prime}, S_{r \theta}{ }^{\prime}, S_{\theta \theta}{ }^{\prime}, S_{\Delta r}{ }^{\prime}, S_{\Delta \theta}{ }^{\prime}$ найти уравнение для расчета такой физически реализуемой и устойчивой матрицы передаточных функций $G_{\mathrm{j} 1}{ }^{s}$, при которой функционал (9) достигает минимума.

Эта задача синтеза также решается методом Винера-Колмогорова, в соответствии с которым необходимо найти условие равенства нулю первой вариации функционала (9) на классе устойчивых и физически реализуемых варьируемых матриц $G_{\mathrm{j} 1}{ }^{s}$.

В соответствии с выбранным методом найдена первая вариация функционала (9):

$$
\begin{gathered}
\delta \dot{e}_{j}^{H}=\frac{1}{2 \pi j} \int_{-j \infty}^{j \infty} \operatorname{tr}\left\{\Phi_{1 j^{*}}^{\mathrm{H}} \dot{R}_{j} \Phi_{1 j}^{H} G_{j 1}^{s}\left(S_{r r}^{\prime}+\tilde{K}_{0}^{-1} S_{r \theta}^{\prime}+S_{\theta r}^{\prime} \tilde{K}_{0^{*}}^{-1}+\tilde{K}_{0}^{-1} S_{\theta \theta}^{\prime} K_{0^{*}}^{-1}\right)+\Phi_{1 j^{*}}^{H} \dot{R}_{j}\left(\Phi_{2 j}^{H} S_{r \Delta}^{\prime}+\Phi_{2 j}^{H} S_{\theta \Delta}^{\prime} \tilde{K}_{0^{*}}^{-1}-\right.\right. \\
\left.\left.-\Phi_{0}^{s} S_{r r}^{\prime}-\Phi_{0}^{s} S_{\theta r}^{\prime} \tilde{K}_{0^{*}}^{-1}\right)\right] \delta G_{j 1^{*}}^{s}+ \\
\delta G_{j 1}^{s}\left[\left(S_{r r}^{\prime}+\tilde{K}_{0}^{-1} S_{r \theta}^{\prime}+S_{\theta r}^{\prime} \tilde{K}_{0^{*}}^{-1}+\tilde{K}_{0}^{-1} S_{\theta \theta}^{\prime} \tilde{K}_{0^{*}}^{-1}\right) G_{j 1^{*}}^{s} \Phi_{1 j^{*}}^{H} \dot{R}_{j} \Phi_{1 j}^{H}+\left(S_{\Delta r}^{\prime} \Phi_{2 j^{*}}^{H}+\right.\right. \\
\left.\left.\left.+\tilde{K}_{0}^{-1} S_{\Delta \theta}^{\prime} \Phi_{2 j^{*}}^{H}-S_{r r}^{\prime} \Phi_{0^{*}}^{s}-\tilde{K}_{0}^{-1} S_{r \theta}^{\prime} \Phi_{0^{*}}^{s}\right) \dot{R}_{j} \Phi_{1 j}^{H}\right]\right\} d s .
\end{gathered}
$$

Если в результате винеровской факторизации [11] найти дробно-рациональную матрицу $\Gamma_{j}^{s}$, устойчивую вместе с обратной и такую, что

$$
\Gamma_{j^{*}}^{s} \Gamma_{j}^{s}=\Phi_{1 j^{*}}^{H} \dot{R}_{j} \Phi_{1 j}^{H},
$$

в результате факторизации найти дробно-рациональную матрицу $D_{\mathrm{j}}^{\mathrm{s}}$ со свойствами аналогичными матрице $\Gamma_{j}^{s}$ при которой выполняется равенство

$$
D_{j}^{s} D_{j^{*}}^{s}=S_{r r}^{\prime}+\tilde{K}_{0}^{-1} S_{r \theta}^{\prime}+S_{\theta r}^{\prime} \tilde{K}_{0^{*}}^{-1}+\tilde{K}_{0}^{-1} S_{\theta \theta}^{\prime} \tilde{K}_{0^{*}}^{-1},
$$

а также выполнить винеровскую сепарацию [10] матрицы $T_{\mathrm{j}}^{\mathrm{s}}$ вида

$$
T_{j}^{s}=T_{j 0}^{s}+T_{j+}^{s}+T_{j-}^{s}=\left(\Gamma_{j^{*}}^{s}\right)^{-1} \Phi_{1 j^{*}}^{H} \dot{R}_{j}\left(\Phi_{2 j}^{H} S_{r \Delta}^{\prime}+\Phi_{2 j}^{H} S_{\theta \Delta}^{\prime} \tilde{K}_{0^{*}}^{-1}-\Phi_{0}^{s} S_{r r}^{\prime}-\Phi_{0}^{s} S_{\theta r}^{\prime} \tilde{K}_{0^{*}}^{-1}\right)\left(D_{j^{*}}^{s}\right)^{-1},
$$

то функционал (19) можно переписать так:

$$
\delta \dot{e}^{H}{ }_{j}=\frac{1}{2 \pi j} \int_{-j \infty}^{j \infty} \operatorname{tr}\left[\Gamma_{j^{*}}^{s}\left(\Gamma_{j}^{s} G_{j 1}^{s} D_{j}^{s}-T_{j}^{s}\right) D_{j^{*}}^{s} \delta G_{j 1^{*}}^{s}+\delta G_{j 1}^{s} D_{j}^{s}\left(D_{j^{*}}^{s} G_{j 1^{*}}^{s} \Gamma_{j^{*}}^{s}-T_{j^{*}}^{s}\right) \Gamma_{j}^{s}\right] d s .
$$

Условие равенства нулю первой вариации (23) на классе устойчивых и физически реализуемых матриц передаточных функций $G_{\mathrm{j} 1}{ }^{s}$ по аналогии с соотвествующим результатом из монографий $[5,10]$ имеет вид

$$
\Gamma_{j}^{s} G_{j 1}^{s} D_{j}^{s}=\left(T_{j 0}^{s}+T_{j+}^{s}\right)
$$

тогда уравнение для расчета такой физически реализуемой и устойчивой матрицы передаточных функций $G_{\mathrm{j} 1}{ }^{s}$, которая обеспечивает равенство нулю первой вариации функционала (23), представляется так

$$
G_{j 1}^{s} \approx\left(\Gamma_{j}^{s}\right)^{-1}\left(T_{j 0}^{s}+T_{j+}^{s}\right)\left(D_{j}^{s}\right)^{-1} .
$$

Анализ выражений (20)-(23) и уравнения (24) показывает, что структура и параметры матрицы передаточных функций оптимального корректирующего устройства при случайных сигналах учитывают свойства возмущений, действующих на систему в том и только в том случае, когда векторы программных сигналов, шумов измерения и возмущений являются взаимосвязанными векторными случайными процессами.

При условии близости вектора сигналов $\dot{u}_{2}^{H}$ входов в объект к одному из векторов входов в объект из набора (1) характеристик объекта по результатам идентификации его моделей динамики дальнейший синтез коррекции в исследуемой системе управления можно прекратить.

Таким образом, задача, поставленная в статье, решена.

Заключение

В работе предложены метод и алгоритм синтеза оптимизированной (оптимальной) структур регулятора в разомкнутой системе стохастического управления многомерным нелинейным объектом. Предложенный метод предлагает последовательность выполнения операций приближения к оптимальной системе путем сравнения характеристик векторов управления и выбора соответствующей модели динамики объекта управления. 
В результате рассмотрения полученных уравнений для синтеза структуры и параметров матриц передаточных функций звеньев коррекции установлено, что разомкнутую систему управления целесообразно применять для нелинейного многомерного объекта, подверженного действию стохастических полезных сигналов, возмущений и помех, если случайные составляющие этих воздействий являются коррелированными.

\section{Литература}

[1] Osadchy S. I. The dynamic characteristics of the mechanism with parallel kinematic structure based on experimental data / S. I. Osadchy, V. A. Zozulya, A. S. Timoshenko // Proceedings of the 7th IEEE International Conference on Intelligent Data Acquisition and Advanced Computing Systems (IDAACS'2013), Berlin, Germany, 2013, vol. 2, pp. $905-911$.

[2] Фельдбаум А.А. Основы теории оптимальных автоматических систем/ Фельдбаум А.А. - М.: Наука, 1966. $623 \mathrm{c}$.

[3] Блохин Л.Н., Житецкий Л.С. Нелинейные оптимальные системы схастической стабилизации // Кибернетика и вычислительная техника. - 2003. - Вып. 139.-С.12-23.

[4] Осадчий С.И. Комбинированный метод синтеза оптимальных систем стабилизации многомерных подвижных объектов при стационарных случайных воздействиях / С.И. Осадчий, В.А. Зозуля. // Международный научнотехнический журнал «Проблемы управления и информатики». - 2013. - №3. - С. 40-49.

[5] Блохин Л.Н. Технологии конструирования современных конкурентоспособных комплексов управления стохастическим движением объектов / Осадчий С.И., Дидык О.К., Рудюк Г.И. - Кировоград: Издатель Лысенко В.Ф., 2015. - 284 с. ISBN 978-617-7197-30-9

[6] Оптимальні та робастні системи керування технологічними об'єктами : монографія / Н.М. Луцька, А.П. Ладанюк - К.: Видавництво Ліра-К, 2015. - 288c.

[7] Osadchiy S.I. Structural Identification of Unmanned Supercavitation Vehicle Based on Incomplete Experimental Data / S.I. Osadchiy, V.M. Kalich, O.K. Didyk // Proceedings of the 2013 IEEE $2^{\text {nd }}$ International Conference "Actual Problems of Unmanned Air Vehicles Developments", Kyiv, 2013, pp. 93-95

[8] Блохін Л.М., Сафронова І.Ю. Новий метод структурної ідентифікації моделей динаміки рухомих об'єктів в штатних режимах функціонування// Веснік ПНЦ ТАУ. -2003, -Вип. 6 - С.60-62.

[9] Гантмахер Ф.Р. Теория матриц/ Ф.Р.Гантмахер -4-е изд.- М.: Наука, 1988.- 552c.

[10] Азарсков В.Н. Методология конструирования оптимальных систем стохастической стабилизации: Монография / В.Н. Азарсков, Л.Н. Блохин, Л.С. Житецкий / Под ред. Блохина Л.Н. - К.: Книжное издательство НАУ, 2006. 437c.

[11] Davis M.C. Factoring the spectral matrix// IEEE Trans. Automat. Cointr. - 1963.- AC-8, N 4. - p. $296-305$.

\section{References}

[1] Osadchy S. I. The dynamic characteristics of the mechanism with parallel kinematic structure based on experimental data / S. I. Osadchy, V. A. Zozulya, A. S. Timoshenko // Proceedings of the 7th IEEE International Conference on Intelligent Data Acquisition and Advanced Computing Systems (IDAACS'2013), Berlin, Germany, 2013, vol. 2, pp. $905-911$.

[2] Feldbaum A.A. Osnovyi teorii optimalnyih avtomaticheskih sistem/ Feldbaum A.A. - M.: Nauka, 1966. - 623s.

[3] Blokhin L.N., Zhytetskyy L.S. Nelyneynye optymal'nye systemy skhastycheskoy stabylyzatsyy // Kybernetyka y vychyslytel'naya tekhnyka. - 2003. - Vyp. 139.-S.12-23.

[4] Osadchiy S.I. Kombinirovannyiy metod sinteza optimalnyih sistem stabilizatsii mnogomernyih podvizhnyih ob'ektov pri statsionarnyih sluchaynyih vozdeystviyah / S.I. Osadchiy, V.A. Zozulya. // Mezhdunarodnyiy nauchnotehnicheskiy zhurnal «Problemyi upravleniya i informatiki». - 2013. - \#3. - S. 40-49.

[5] Blokhin L.N. Tekhnolohyy konstruyrovanyya sovremennykh konkurentosposobnykh kompleksov upravlenyya stokhastycheskym dvyzhenyem ob"ektov / Osadchyy S.Y., Dydyk O.K., Rudyuk H.Y. - Kyrovohrad: Yzdatel' Lysenko V.F., 2015. - 284 s. ISBN 978-617-7197-30-9

[6] Optymal'ni ta robastni systemy keruvannya tekhnolohichnymy ob"yektamy : monohrafiya / N.M. Luts'ka, A.P. Ladanyuk - K.: Vydavnytstvo Lira-K, 2015. - 288s.

[7] Osadchiy S.I. Structural Identification of Unmanned Supercavitation Vehicle Based on Incomplete Experimental Data / S.I. Osadchiy, V.M. Kalich, O.K. Didyk // Proceedings of the 2013 IEEE $2^{\text {nd }}$ International Conference "Actual Problems of Unmanned Air Vehicles Developments", Kyiv, 2013, pp. 93-95

[8] Blokhin L.M., Safronova I.YU. Novyy metod strukturnoyi identyfikatsiyi modeley dynamiky rukhomykh ob"yektiv v shtatnykh rezhymakh funktsionuvannya// Vesnik PNTS TAU. -2003, -Vyp. 6 - S.60-62.

[9] Gantmaher F.R. Teoriya matrits/ F.R.Gantmaher -4-e izd.- M.: Nauka, 1988.- 552s.

[10] Azarskov V.N. Metodologiya konstruirovaniya optimalnyih sistem stohasticheskoy stabilizatsii: Monografiya / V.N. Azarskov, L.N. Blohin, L.S. Zhitetskiy / Pod red. Blohina L.N. - K.: Knizhnoe izdatelstvo NAU, 2006. - 440s.

[11] Davis M.C. Factoring the spectral matrix// IEEE Trans. Automat. Cointr. - 1963.- AC-8, N 4. - p. $296-305$. 